\title{
Molecular Dynamic simulations of diffusion in the clay matrix
}

\author{
THOMAS R. UNDERWOOD", IAN C. BOURG' \\ Civil and Environmental Engineering, \\ Princeton University, NJ, 08544 \\ (*correspondance: thomas.underwood@ princeton.edu)
}

Fine-grained sediments and sedimentary rocks play important roles in a variety of modern energy technologies from petroleum geology to geological carbon sequestration and radioactive waste management. However, despite their utility and ubiquity, many of their properties remain poorly understood. In particular, the ability to predict the permeability and mechanics of these media remains a persistent fundamental challenge in the geosciences.

In the present work, we show how large-scale classical molecular dynamics (MD) simulations can help interpret the properties of fine-grained sedimentary material. All-atom simulations containing 30 discrete clay particles are utilized to understand the evolution of a clay nanoparticle suspension during its progressive dehydration. Microstructural (pore size distribution, tortuosity, anisotropy), thermodynamic (enthalpy and free energy of hydration, anion exclusion), mechanical (total suction), and transport properties (diffusion coefficient tensors of water and sodium) are calculated and compared to the experiment.

The presented results provide new insight into the coupled chemistry, mechanics, and transport properties of disordered nanoparticle assemblages and shed light upon the important role of water films in controlling these properties. 